\title{
The shape of you: do individuals associate particular geometric shapes with identity?
}

\author{
Valerio Manippa ${ }^{1}$ (D) $\cdot$ Luca Tommasi ${ }^{1}$
}

Accepted: 4 September 2021

(C) The Author(s) 2021

\begin{abstract}
For more than a century, psychologists have been interested in how visual information can arouse emotions. Several studies have shown that rounded shapes evoke positive feelings due to their link with happy/baby-like expressions, compared with sharp angular shapes, usually associated with anger and threatening objects having negative valence. However, to date, no-one has investigated the preference to associate simple geometric shapes to personal identities, including one's own, that of a close acquainted, or that of a stranger. Through 2 online surveys we asked participants to associate a geometric shape, chosen among a circle, a square and a triangle, to each of three identities, namely "you" (the self), "your best friend" or "a stranger". We hypothesized that the circle would be more associated with the self, the square with the friend and the triangle with the stranger. Moreover, we investigated whether these associations are modulated by 3 personality traits: aggressivity, social fear and empathy. As predicted, we found that participants associate more often the circle with the self, both the circle and the square with the best friend, whereas they matched angular shapes (both the triangle and the square) to the stranger. On the other hand, the possibility that personality traits can modulate such associations was not confirmed. The study of how people associate geometric figures with the self or with other identities giving them an implicit socio-affective connotation, is interesting for all the disciplines interested in the automatic affective processes activated by visual stimuli.
\end{abstract}

Keywords Geometric form $\cdot$ Self $\cdot$ Personality $\cdot$ Perception $\cdot$ Aesthetics $\cdot$ Preference

\section{Introduction}

Even before the foundation of empirical aesthetics as an academic discipline, the English painter William Hogarth argued that S-shaped, or serpentine, lines, which he called "the lines of beauty", are more productive of beauty and lively ornamentation, because they can vary both in length and in degree of curvature, whereas straight lines vary only in length (Corradi \& Munar, 2019; Hogarth, 1753). The study of how more complex stimuli (composed of combinations of straight lines, curved lines and angles) are perceived from an affective point of view, has been deepened two and a half centuries after Hogarth: several evidence have shown that biological and affective cues, such as emotional faces, (Gronau et al., 2003; Sui \& Liu, 2009; Vuilleumier, 2005) capture attention more

Valerio Manippa

valerio.manippa@unich.it

1 Department of Psychological, Health and Territorial Sciences, University "G. d'Annunzio" of Chieti-Pescara, 66100 Chieti, Italy than do most common stimuli without a biological or affective relevance (e.g., Ro et al., 2001). For example, emotionallycharged expressions and baby faces draw attention more than neutral faces (Brosch et al., 2007; Palermo \& Rhodes, 2007), whereas other stimuli can also strongly grasp our attention when present in a crowd, including knives, guns, syringes and dangerous animals (e.g., snakes, spiders), namely negative/threatening stimuli which require a rapid response. The ability to easily recognize the geometric patterns (i.e., lines, curves and angles) associated to such dangerous or rewarding objects has deep evolutionary meaning, since all these items automatically arouse specific emotions and behaviors that are crucial for survival (Gable \& Harmon-Jones, 2008; Ohman \& Mineka, 2001).

To date, we can assert that research has made clear that humans prefer curved shapes to their angular counterparts: several studies (e.g., Silvia \& Barona, 2009; Watson et al., 2012; Bar \& Neta, 2006) have shown that curved lines and symmetrical shapes are preferred given their association with the happiness expression and the infant face, containing more curvilinear elements. The same studies have suggested that angularities and asymmetrical shapes are disliked, due to their 
association with the anger expression and dangerous objects such as knives. Moreover, in 1993 Ekman found the presence of cross-cultural regularities in facial displays of emotion: anger and happiness masks used in ritual functions of different cultures, revealed that a set of geometric patterns, rather than actual facial features, convey different emotional meanings (Aronoff et al., 1992). A further advancement was made by Aronoff (2006), who demonstrated the power of nonrepresentational visual patterns to produce meaning by presenting materials that included geometric shapes in a variety of line drawings, large-scale physical movement in classical ballet (e.g., body displays and arm displays) and spatial relationships among individuals portrayed in seventeenth century Dutch art. Results across all these studies suggested that at least for the emotions of anger and happiness, meaning is conveyed by specific geometric properties of the visual display, angularities being more likely associated with anger and roundness with happiness.

The study of the relationship between semantic/expressive aspects and geometric shapes, started almost one century ago, with the pioneering researches on "sound symbolism" (Köhler, 1970; Sapir, 1929), namely the implicit association between visual stimuli (i.e. shapes) and words/syllables (D'Anselmo et al., 2019; Westbury, 2005). Later, in 1957, Osgood and coworkers asked to evaluate some geometric patterns on a set of subjective semantic differential scales and indicated the degree of "badness", "potency" and "activity" of each visual stimulus (Osgood et al., 1957). It turned out that whereas sharp-angled shapes conveyed negative meaning, rounded shapes elicited positive attitudes (Aronoff et al., 1992). Similar results were found by Bar and Neta (2006) using a forced choice 'liking' task: participants disliked neutral objects comprised of pointed features and sharp angles significantly more than curved ones (e.g., a watch with a sharp-angled contour in comparison with one with a curved contour). Similarly, Pavlova et al. (2005) found a positive correlation between negative emotions and the perceived instability of geometric shapes such as the triangle and the oval.

Curiously, in 2012 Sui and coworkers developed a novel associative learning approach aimed at demonstrating the stronger salience of self-relevant over non-self-relevant features. Various geometric shapes (i.e., circle, square and triangle) were associated by the experimenter to a label indicating the self (e.g., "you"), a familiar other (e.g., "friend") or an unfamiliar other (e.g., "stranger"). Then participants had to judge, in a rapid presentation task, whether the shape and the label were correctly matched. A substantial advantage in terms of faster reaction times and higher accuracy was found when participants identified the shape (whatever it was) associated with the self, relative to non-self-matched pairs. They called this advantage "self-prioritization benefit" and proposed that self-associated shapes automatically engage the reward system increasing attention.
However, Sui et al. (2012) did not assess whether associating one or another shape to the various identities played any role on their dependent variables. In fact, considering the preference and the positive attitudes associated with curvilinearity, one might wonder whether the RTs and accuracy observed when identifying the circle-self pair would have been respectively faster and better compared with those observed when identifying the triangle-self pair. Similarly, it might also be that the stranger-triangle match could be easier to recognize since the triangle might implicitly convey some sort of threat by means of its sharp angles. The square, that is neither positive and rounded as the circle nor sharped and negative as the triangle, might be the geometric shape allowing for the most appropriate congruency when matched with an intermediate identity in between self and stranger, as is the friend.

To summarize, for more than a century psychologists have been interested in how individuals associate visual information with emotional valence (Barrett \& Bar, 2009; Lundholm, 1921), showing that rounded shapes evoke positive feelings due to their link with happy/infant-like expression, compared with sharp/angular shapes, usually associated with the anger expression and threatening objects having negative valence (Bar \& Neta, 2006; Carbon, 2010; Leder \& Carbon, 2005; Silvia \& Barona, 2009). However, to the best of our knowledge, no investigation has been carried out to date to assess the preference to associate simple geometric shapes to specific individual identities. In this study, we asked our participants to associate a geometric shape amongst the circle, the square and the triangle, to only one identity, namely "you" (the self), "a friend" or "a stranger". Our main hypotheses $(H)$ were:

\section{Hla: Individuals are more likely to associate the circle with the self; \\ H1b: Individuals are more likely to associate the triangle with the stranger;}

Since the best friend is neither "positive" as the self (circle) nor "threatening" as the stranger (triangle) we also hypothesize that:

H1c: Individuals are more likely to associate the square with the best friend;

We also had 3 further hypotheses based on personality traits. In fact, if the link between the valence associated to geometric patterns depends on the implicit socio-affective meaning that such shapes convey, we predicted that personality traits such as aggressivity (i.e., an aggressive attitude), social phobia (i.e., an attitude to see strangers as dangerous) and empathy (i.e., an attitude to share emotions) could influence the geometric shape that participants decide to associate with identities. 
For example, individuals who describe themselves as more aggressive, could be more prone to associate the triangle to the self (the more threatening shape) compared with less aggressive individuals. Also, social phobic individuals would see the stranger as extremely threatening compared with individuals scoring lower in social phobic traits, hence the former could associate the triangle to the stranger more often compared with the latter. Last, more empathic individuals, who are more able to feel the same experience that another is feeling, could be more prone to share the circle (the more positive shape) with their best friend, maybe for the stronger experience/emotional sharing compared with less empathic individuals. Hence, we hypothesized that:

H2a: Aggressive individuals are more likely to associate the triangle with the self, compared with less aggressive ones;

H2b: Social phobic individuals are more likely to associate the triangle with the stranger, compared with less social phobic ones,

H2c: Empathic individuals are less likely to associate the circle to the self/more likely to associate the circle to the friend compared with lesser empathic ones.

\section{Experiment 1}

In this experiment, besides the key question regarding the shape associated with the self, the friend or the other, we asked participants to fill in 3 questionnaires to assess whether aggressivity, social fear and empathy influence the shape associated with the 3 identities. We expected that participants selfreporting higher aggressivity traits could associate the triangle more often with the self, due to the implicit association of this geometric shape with dangerous stimuli (Wrangham, 2018). On the other hand, we expected that individuals with high social fear would associate the triangle to the stranger more often compared to individuals with lower social fear, less prone to judge the stranger as dangerous (Stopa et al., 2013). Finally, we predicted that empathic people would more likely share the circle with the friend, compared with less empathic individuals (e.g., Eisenberg \& Fabes, 1990).

\section{Materials and Methods}

\section{Participants}

Three hundred forty-nine Italian participants responded to internet advertisements and filled in the survey. Of them, 236 (201 female) filled in the survey correctly, namely associating each and every shape with a single and exclusive identity, and vice versa (e.g., participants that associated the square with both the "self" and the "friend" were excluded). Participants were mainly university students $(N=126)$ with a $M$ age of $26.7(S D=7.1)$ and the majority of them were right-handed $(N=203)$.

\section{Procedure}

The survey (written in Italian) was developed and administered via Google Forms and it was composed of 6 sections. The first and second sections consisted, respectively, of the informed consent and a socio-demographic questionnaire (age, sex, employment, handedness). The third section was the shape-identity association task: specifically, these instructions were shown: "You have to indicate which geometric shape among those shown below you would associate with yourself, with your best friend and with a stranger. There is only one rule to respect: a geometric shape can be associated with a single identity (you cannot associate the same shape with two different identities)". Then 3 questions appeared: "Which of these shapes is you? / Which of these shapes is your best friend / Which of these shapes is a stranger?'. Below each question, a circle, a square and an equilateral triangle were displayed and only one of each triplet could be selected with a mouse click (see Fig. 1). Both the order of the questions and of the geometric shapes were randomized. After this section participants filled the Italian versions of the Aggression Questionnaire (Buss \& Perry, 1992; Fossati et al., 2003) in section 4, of the Balanced Emotional Empathy Scale (Mehrabian, 1996; Meneghini et al., 2006) in section 5 and of the Social Fear Scale (Raulin \& Wee, 1984) in section 6.

\section{Questionnaires}

The Aggression Questionnaire (AGQ; Buss \& Perry, 1992) is a self-report scale designed to measure four major components of aggressivity (physical aggressivity, verbal aggressivity, anger and hostility) and consists of 29 items which are rated on a 7-point Likert scale ranging from 1 (extremely uncharacteristic of me) to 7 (extremely characteristic of me). In this study the value of Cronbach's alpha was 0.879 .

The Social Fear Scale (SFS; Raulin \& Wee, 1984) is a selfreport questionnaire assessing the responder's social phobia. It consists of 36 items with a dichotomous response (true-false) aimed at measuring the avoidance of interpersonal relationships, social inadequacy and the scarcity of social relationships. In this study the value of Cronbach's alpha was 0.853 .

The Balanced Emotional Empathy Scale (BEES; Mehrabian, 1996) is a self-report questionnaire assessing emotional empathy, defined as an individual's tendency to vicariously share other people's emotions. It consists of 30 items, in which participants are asked to express their degree of agreement to each of the 30 statements comprising the instrument, on a 7-point Likert scale, with values ranging from 


\section{Shape-Identity}

You have to indicate which geometric shape among those shown below you would associate with yourself, your best friend and a stranger.

There is only one rule to respect: a geometric shape can be associated with a single identity (you cannot associate the same shape with two different identities)

Which of these shapes is you?

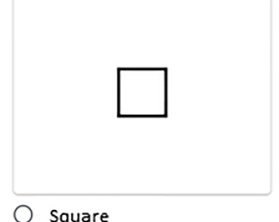

Square
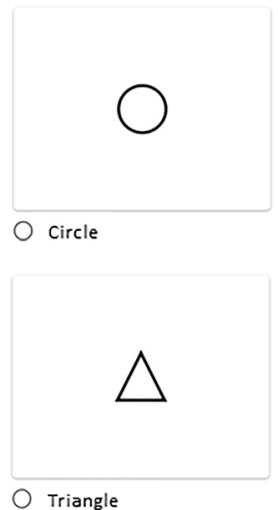

Fig. 1 The third section of the survey, in which participants were asked to choose a geometric shape to associate with the self, the best friend and the stranger (shape-identity association task). Both the order of the questions

+3 ("completely agree") to -3 ("completely disagree"). In this study the value of Cronbach's alpha was 0.851 .

In all cases higher scores are associated with stronger traits (e.g., high scores in AGQ describe a more aggressive individual).

\section{Results and Discussion}

Data analyses were carried out using IBM SPSS Statistics version 20. First of all, for descriptive purpose we analyzed the correlations amongst the scores of the 3 questionnaires: we found a positive correlation between the AGQ score and the SFS score $(r=0.3735, p<0.001)$ and no other correlation (see the Table 1).

Then, we carried out 3 Chi-square tests to evaluate whether the geometric shapes (Circle, Square, Triangle) were associated with the 3 identities (Self, Friend, Stranger) differently from what could be expected from chance. We found that the shapes associated with the Self $\left(\chi 2_{(2)}=14.246, p=0.001\right)$ and with the Stranger $\left(\chi 2_{(2)}=39.941, p<0.001\right)$ were statistically different from chance (see Fig. 2), but the same was not true for the Friend. Pairwise comparisons (statistical significance set at $p<0.012$ due to the Bonferroni multiple
Which of these shapes is a stranger?
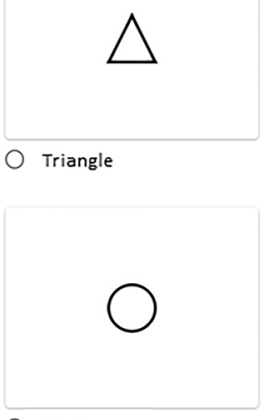

Circle

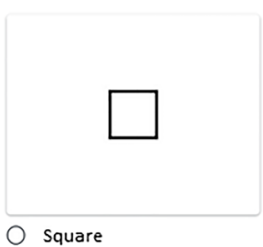

(identities) and the order of the response alternatives (geometric shapes) were randomized

comparisons correction) showed that the shape significantly more associated to the Self is the Circle, compared with both the Square $\left(\chi 2_{(1)}=9.830, p=0.002\right)$ and the Triangle $(\chi$ $\left.2_{(1)}=9.830, p=0.002\right)$. On the contrary, the Circle was the shape less associated with the Stranger compared with both the Square $\left(\chi 2_{(1)}=22.781, p<0.001\right)$ and the Triangle $(\chi$ $\left.2_{(1)}=34.766, p<0.001\right)$. No other significant pairwise comparison was observed. All the data are reported in Table 2.

These findings confirmed our hypotheses $H 1 a$ and $H 1 b$. Particularly, as predicted by hypothesis $H 1 a$, individuals are

Table 1 Correlations between the scores of the questionnaires used in Experiment 1. AGQ = Aggressive Questionnaire, SFS = Social Fear Scale, BEES = Balanced Emotional Empathy Scale. Asterisks indicate significant correlations

\begin{tabular}{|c|c|c|c|c|c|c|}
\hline & \multicolumn{2}{|l|}{ AGQ } & \multicolumn{2}{|l|}{ SFS } & \multicolumn{2}{|l|}{ BEES } \\
\hline & $R$ & $p$ & $R$ & $p$ & $R$ & $p$ \\
\hline AGQ & & & .3185 & $<.001 *$ & -.0935 & $=.350$ \\
\hline SFS & .3185 & $<.001 *$ & & & -.0142 & $=.152$ \\
\hline BEES & -.0935 & $=.35$ & -.0142 & $=.152$ & & \\
\hline
\end{tabular}



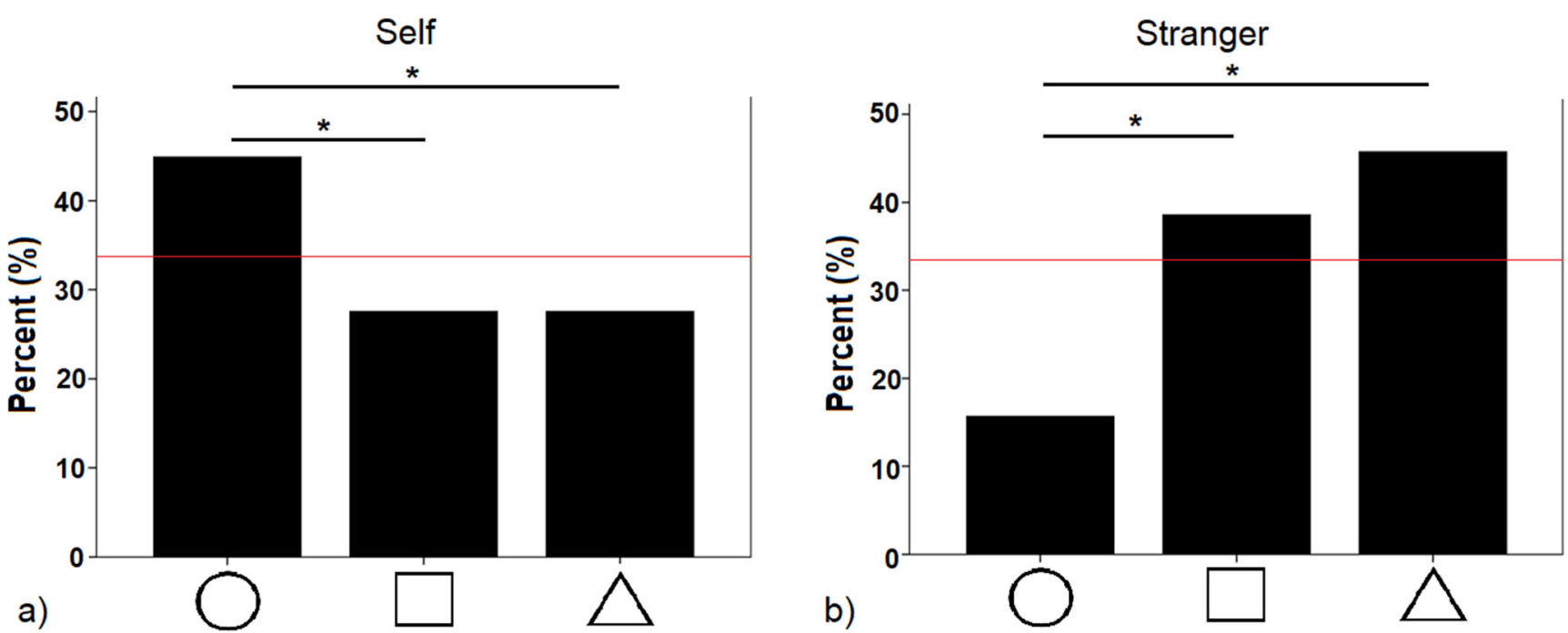

Fig. 2 Association of each geometric shape with the Self and the Stranger, expressed in percentage. Red lines indicate chance level, asterisks indicate significant pairwaise comparisons. $(p<0.012)$

more likely to associate the circle to the self, possibly because of the positive valence associated to curved shapes compared to angular shapes and their association with dangerous entities. On the contrary, the circle is the shape less frequently associated to the stranger, whereas the angular shapes were associated with this identity, partially confirming hypothesis $H 1 b$, since there was no difference between the triangle and the square. Regarding the shape associated to the friend $(H 1 \mathrm{c})$, participants showed no significant preference.

We carried out 3 further Chi-square tests to evaluate whether the assessed personality traits determined a different shapeidentity matching. With this aim, we divided our sample according to the median score to each questionnaire, obtaining 2 groups for each independent variable (Low Aggressivity vs. High Aggressivity, Low Social Fear vs. High Social Fear and Low Empathy vs. High Empathy). We did not find any significant interaction between the shape-identity associations and the assessed personality traits.

Hence, contrary to our predictions, the shape-identity matching was not influenced by aggressivity, social fear and empathy of our participants, disconfirming our Hypotheses 2.

Table 2 The frequency of association of each geometric shape (Circle, Square and Triangle) to each identity (Self, Friend and Stranger) reported as observed frequency $(N)$ and in percentage $(\%)$ for Experiment 1

\begin{tabular}{|c|c|c|c|c|c|c|c|c|}
\hline & \multicolumn{2}{|l|}{ Self } & \multicolumn{2}{|c|}{ Friend } & \multicolumn{2}{|c|}{ Stranger } & \multicolumn{2}{|c|}{ Chance } \\
\hline & $N$ & $\%$ & $N$ & $\%$ & $N$ & $\%$ & $N$ & $\%$ \\
\hline Circle & 106 & 44.9 & 93 & 39.4 & 37 & 15.7 & 78.7 & 33.3 \\
\hline Square & 65 & 27.5 & 80 & 33.9 & 91 & 38.6 & 78.7 & 33.3 \\
\hline Triangle & 65 & 27.5 & 63 & 26.7 & 108 & 45.8 & 78.7 & 33.3 \\
\hline
\end{tabular}

\section{Experiment 2}

In this experiment we assessed again which geometric shape is more likely associated with the three identities, and whether personality traits can influence such a matching. However, in this experiment we assessed personality using a broaderbandwidth tool, the Italia Personality Inventories (Perussia, 2006), a questionnaire specifically validated for the Italian population. Such inventory evaluates 7 personality traits/factors: dynamism, vulnerability, empathy, conscientiousness, imagination, defensiveness, introversion and was chosen for 2 main reasons: i) the Italia Personality Inventories is developed and validated for the Italian population, which our sample belongs to; ii) some of the personality traits assessed by this inventory are those already assessed in the previous experiment, but the inventory is not limited to them. In this way we wished to confirm the tendency to associate the self with the circle and the stranger with the angular shapes but wanted to deepen the possible interactions between shape-identity matching and personality traits related to aggressivity, social fear and empathy using another self-report instrument.

\section{Materials and Methods}

\section{Participants}

Four hundred thirty-nine Italian participants responded to internet advertisements and filled in the survey. Of them, 326 (256 female) filled in the survey correctly, namely associating each and every shape with a single and exclusive identity, and vice versa (e.g., participants that associated the square with both the "self" and the "friend" were excluded). Participants were mainly university students $(N=224)$ with $M$ age of 27.6 
$(S D=8.0)$ and the majority of them were right-handers $(N=$ 290).

\section{Procedure}

The procedure and the survey were the same as described in Experiment 1 but, in this case, the three last sections in which the AGQ, the BEES and the SFS were presented, were replaced by one section in which the General Italia Personality Inventory (Perussia, 2006) was presented.

\section{Questionnaire}

The General (or Great) version of the Italia Personality Inventory (ITAPI-G) consists of 105 items, 15 items for each of the seven traits-factors which are: i) Dynamism (i.e., resourcefulness, curiosity, vivacity); ii) Vulnerability (i.e., discomfort, fear, suffering); iii) Empathy (i.e., solidarity, sociability, sensitivity); iv) Conscientiousness (i.e., perseverance, precision, rationality); v) Imagination (i.e., creativity, feeling, fantasy); vi) Defensiveness (i.e., rigidity, materiality) and vii) Introversion (i.e., introspection, self-sufficiency, isolation). For each statement, participants are asked to report their degree of agreement on a 4-points Likert scale ranging from 1 (completely disagree) to 4 (completely agree). The higher is the score of a specific trait, the stronger is that specific trait. In this study the value of Cronbach's alpha of each factor's subscale ranged from 0.819 to 0.893 .

As anticipated, the choice of ITAPI-G was determined by two reasons: i) such inventory was developed and validated in the Italian population, which our sample represents; ii) we supposed that several factors assessed through the ITAPI correlate with the personality traits of interest for our study (aggressivity, empathy and social phobia) and would thus be suitable to deepen our H2. To better check such assumption, before carrying out Experiment 2 the ITAPI-G, AGQ, SFS and BEES were administered via Google Forms to an additional and independent sample of 102 participants (90 females, $M$ age $=30.8, S D=9.8)$. Then we tested the correlations between the scores of the AGQ, SFS and BEES and the scores of the 7 factors of the ITAPI-G. All the correlations are resumed in Table 3. In particular, the AGQ score is positively correlated with the Defensiveness and the Vulnerability scores (both $p<0.001$ ) and negatively correlated with the Empathy score $(p<0.001)$. The SFS score is positively correlated with the Vulnerability, Defensiveness and Introversion scores (all $p<0.001)$. Finally, the BEES score is positively correlated with the Empathy $(p<0.001)$ and Imagination scores $(p<$ 0.008 ) and negatively correlated with the Introversion score ( $p=0.036)$. Dynamism and Consciousness were not related with any trait of interest, and their potential effect on shapeidentity matching was anyhow investigated for explorative purposes.

\section{Results and Discussion}

Data analyses were carried out using IBM SPSS Statistics version 20. First, we carried out 3 Chi-square tests to evaluate whether the geometric shapes (Circle, Square, Triangle) were associated with the identities (Self, Friend, Stranger) differently from chance. We found that the shapes associated with the Self $\left(\chi 2_{(2)}=17.933, p<0.001\right)$, with the Friend $\left(\chi 2_{(2)}=\right.$ $16.276, p<0.001)$ and with the Stranger $\left(\chi 2_{(2)}=53.822, p\right.$ $<0.001$ ) were all statistically different from chance (see Fig. 3). Moreover, pairwise comparisons (statistical significance set at $p<0.012$ due to the Bonferroni multiple comparisons correction) showed that the shape significantly more associated to the Self was the Circle, compared with both the Square $\left(\chi 2_{(1)}=16.538, p<0.001\right)$ and the Triangle $\left(\chi 2_{(1)}\right.$ $=7.230, p=0.007)$. Regarding the Friend, both the Circle $(\chi$ $\left.2_{(1)}=16.343, p<0.001\right)$ and the Square $\left(\chi 2_{(1)}=6.821, p=\right.$ $0.009)$ were significantly more associated to this identity compared with the Triangle. Finally, the Triangle $\left(\chi 2_{(1)}=53.313\right.$, $p<0.001)$ and the Square $\left(\chi 2_{(1)}=39.640, p<0.001\right)$ were the shapes significantly more associated to the Stranger compared with the Circle. No other significant pairwise comparison was observed. All the data are reported in Table 4.

These findings confirm and extend those of Experiment 1. More specifically, as previously observed, the shape that individuals associate more often with the self is the circle. Regarding the stranger, although with no difference between the triangle and the square, the angular shapes were more often associated to it, with respect to the circle. Interestingly, with a novel and larger sample, an effect emerged also for the friend identity: the triangle was the shape less often associated to the participant's best friend compared with both the circle and the square. Such result did not completely match with our hypothesis $H 1 \mathrm{c}$ but can be easily explained: we asked, "Which of these shapes is your best friend?" and, being the best friend someone you can trust, the choice to avoid the triangle could reflect the individual's perception of the low threat associated to his/her best friend.

In order to assess whether the 7 personality traits influenced the shape-identity association, we divided our sample according to the median score to each trait, obtaining 2 groups for each independent variable (Low Dynamicity vs. High Dynamicity, Low Vulnerability vs. High Vulnerability, Low Empathy vs. High Empathy, Low Conscientiousness vs. High Conscientiousness, Low Imagination vs. High Imagination, Low Defensiveness vs. High Defensiveness and Low Introversion vs. High Introversion).

Again, contrary to our Hypotheses 2, the shape-identity matching was not influenced by any of the ITAPI-G factors. Therefore, also assessing the personality traits we were interested in by means of another instrument, we did not find any interaction between these latter and our identity-shape matching task. 
Table 3 Correlation between the score of the questionnaires used in Experiment 1 and the scores of the 7 factors of the ITAPI-G, used in Experiment 2. Asterisks indicate significant correlations

\begin{tabular}{|c|c|c|c|c|c|c|}
\hline \multirow[b]{2}{*}{ ITAPI-G factors } & \multicolumn{2}{|c|}{ Aggression Questionnaire } & \multicolumn{2}{|c|}{ Social Fear Scale } & \multicolumn{2}{|c|}{ Balanced Emotional Empathy Scale } \\
\hline & $R$ & $p$ & $R$ & $p$ & $R$ & $P$ \\
\hline Dynamicity & .0277 & $=.782$ & -.1698 & $=.088$ & .1168 & $=.242$ \\
\hline Vulnerability & .5800 & $<.001^{*}$ & .3827 & $<.001 *$ & .0666 & $=.506$ \\
\hline Empathy & -3441 & $<.001^{*}$ & -.1351 & $=.176$ & .5182 & $<.001 *$ \\
\hline Consciousness & -.1230 & $=.218$ & .0342 & $=.733$ & .1392 & $=.163$ \\
\hline Imagination & .1771 & $=.075$ & .1756 & $=.078$ & .2607 & $=.008^{*}$ \\
\hline Defensiveness & .5626 & $<.001 *$ & .2617 & $=.008^{*}$ & -.0149 & $=.882$ \\
\hline Introversion & -.1921 & $=.053$ & .4006 & $<.001 *$ & -.2079 & $=.036^{*}$ \\
\hline
\end{tabular}

\section{General Discussions}

Our Hypotheses 1 were substantially confirmed, the results appearing to be congruent with the predictions one can make

\section{Self}

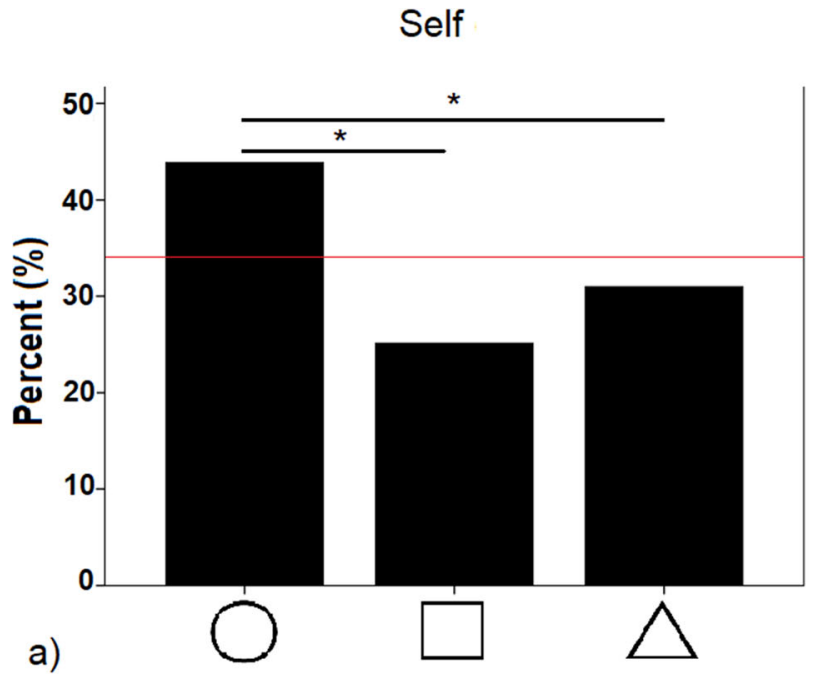

based on the relevant literature. Our participants associated more often the self with the circle, and the best friend with the circle and the square, whereas they chose the angular shapes (both the triangle and the square) to represent the
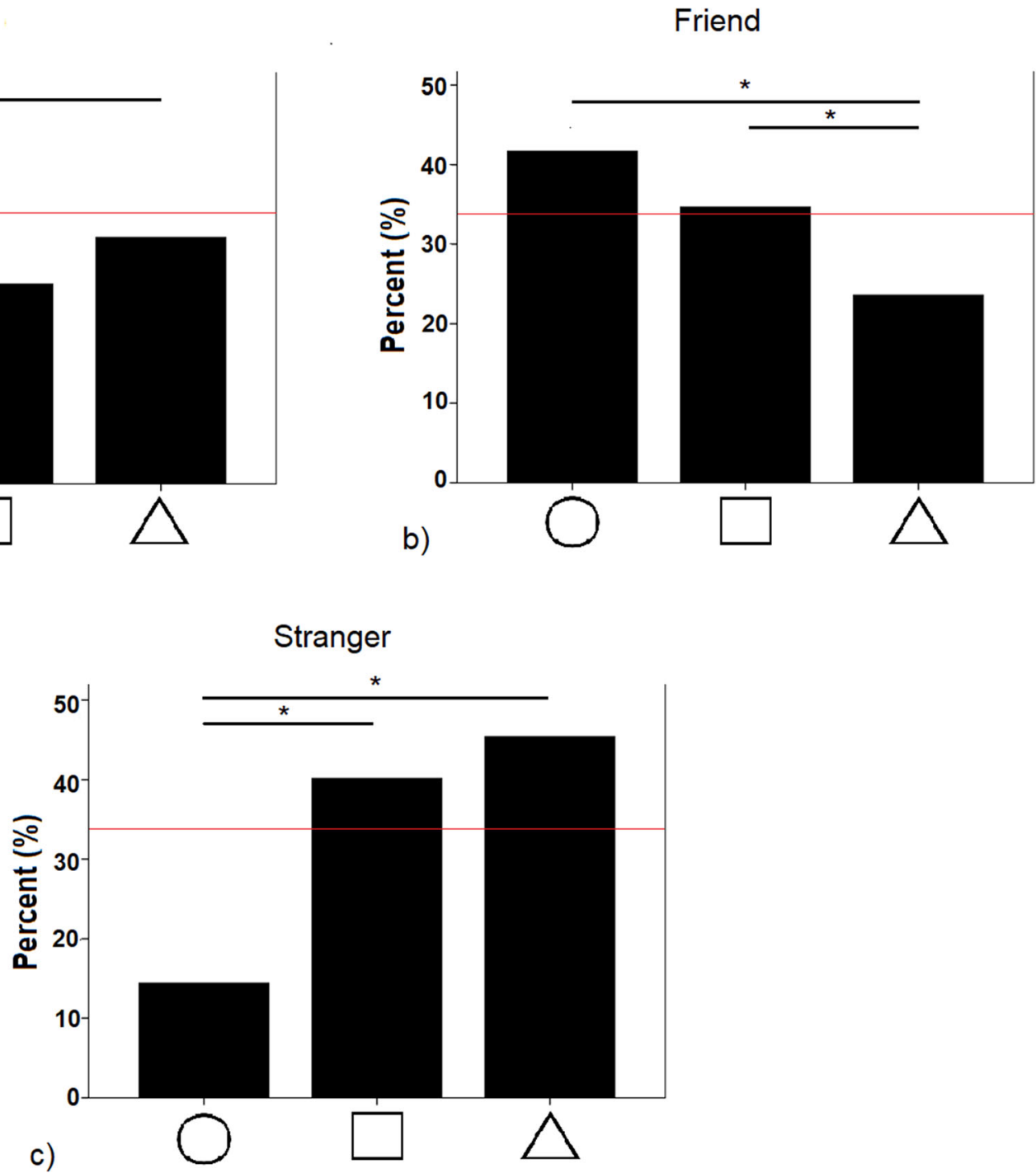

Fig. 3 Association of each geometric shape with the Self, the Friend and the Stranger, expressed in percentage. Red lines indicate chance level, asterisks indicate significant pairwise comparisons. $(p<0.012)$ 
Table 4 The frequency of association of each geometric shape (Circle, Square and Triangle) to each identity (Self, Friend and Stranger) reported as observed frequency $(N)$ and in percentage $(\%)$ for the Experiment 2

\begin{tabular}{|c|c|c|c|c|c|c|c|c|}
\hline & \multicolumn{2}{|l|}{ Self } & \multicolumn{2}{|c|}{ Friend } & \multicolumn{2}{|c|}{ Stranger } & \multicolumn{2}{|c|}{ Chance } \\
\hline & $N$ & $\%$ & $N$ & $\%$ & $N$ & $\%$ & $N$ & $\%$ \\
\hline Circle & 143 & 43.9 & 136 & 41.7 & 47 & 14.4 & 108.7 & 33.3 \\
\hline Square & 82 & 25.2 & 113 & 34.7 & 131 & 40.2 & 108.7 & 33.3 \\
\hline Triangle & 191 & 31.0 & 77 & 23.6 & 148 & 45.4 & 108.7 & 33.3 \\
\hline
\end{tabular}

stranger. On the other hand, the possibility that personality traits modulate such associations (Hypotheses 2) was not confirmed.

Biases toward visual objects can be induced not only by their semantic meaning (e.g., food, or cutting objects) but also by low-level perceptual properties: we can associate a positive or negative valence to an object (also presented as a picture) with a single gaze, depending on the presence of sharp-angled or curved features. For example the group of Velasco and Salgado-Monteiro, reported that positive words and judgments are more often associated with rounded shapes and negative ones with angular ones (Salgado Montejo et al., 2015; Velasco et al., 2016). In line with this literature, we confirmed (Hla) that individuals choose the circle rather than the angular shapes to represent themselves. On the other hand, we proposed $(\mathrm{H} 2 \mathrm{a})$ that aggressive individuals, namely individuals that self-report enacting toward others with the intention to cause them psychological or physical harm (Huesmann, 1994), would have preferred to associate the triangle with the self, due to its link with threatening object shapes and with angry facial expressions (Aronoff, 2006; Osgood et al., 1957). This effect did not occur, neither when we divided our sample according to the score of the AQ (Buss \& Perry, 1992) nor when we used the Vulnerability and the Defensiveness factors (both positively correlated with the AQ score) of the ITAPI-G (Perussia, 2006). We point out that we preferred to use an upward-pointing equilateral triangle instead of a downward-pointing one, to be consistent with the other 2 classical geometric shapes, since also the typicality of a form can play a role in its associated valence (Reber et al., 2004). Despite that, previous research has often demonstrated that, although angular shapes are liked less than curved ones, the downward-pointing triangle, maybe due to the link with the shape drawn by the eyebrows when we are angry, is further disliked and associated with threat (e.g., Larson et al., 2012; Ro et al., 2001). Hence the equilateral triangle, although negatively valenced, could be not so "threatening" to the point of becoming associated with the self-reported aggressivity of our sample.

Probably for the same reason, we found no difference between the angular shapes associated with the stranger and no interaction with the personality traits of social phobia, as assessed with the SFS in Experiment 1 (Raulin \& Wee, 1984) and with the Vulnerability and Introversion factors of the ITAPI-G in Experiment 2 (Perussia, 2006). Particularly, we had predicted that the triangle would be matched more frequently to the stranger compared with the other shapes $(H 1 b)$, being it the most threatening among the identities proposed. Although the circle was the shape less matched with the stranger, there was no difference in the frequencies which the triangle and the square were associated to this identity. We had also predicted that such matching would be further stronger for social phobic individuals $(H 2 b)$, since social phobia is defined as the persistent fear of one or more social situations in which a person is exposed to unfamiliar people. Particularly, the phobic person fears that he/she will act in a way or show anxiety symptoms that will be embarrassing and humiliating due to the stranger response/judgment (Bögels et al., 2010). Such fear for the stranger did not result, as predicted, in an increased triangle-stranger matching.

Finally, we had proposed that the square, which has not a clear emotional connotation compared to the other 2 shapes, would be more frequently associated with the friend $(H I c)$, being this identity not positive as the self but also not negative as the stranger. Whereas in Experiment 1 we found no significant differences, with the larger sample of Experiment 2 we found a preference to associate both the circle and the square with the friend, as compared to the triangle. This finding can be easily explained by the peculiar question that we had formulated: "Which of these shapes is your best friend?". Obviously for most of us, the best friend elicits positive attitudes, thus deserving to be associated with the circle, other than with the square. On the contrary, one's best friend certainly would not be a threat, and this could be the reason why the triangle was less matched with this identity. This explanation can hold true also regarding the lack of evidence supporting hypothesis $H 2 c$, in which we had predicted a role of empathy in shape-identity matching. Empathy refers to sensitivity to, and understanding of, the mental states of others. From an emotional point of view it is "an affective response more appropriate to someone else's situation than to one's own" (Hoffman, 1987) or, as affirmed by Eisenberg and Strayer (1987) "an emotional response that stems from another's emotional state or condition and that is congruent with the other's emotional state or situation". Such statement led us to hypothesize that more empathic individuals would be more prone to "share" the circle with the friend, in a similar manner they shared affective feelings due to their positive relationship. We did not observe such effect independently of the instrument used to assess empathy (i.e., the BEES in Experiment 1 and the empathy factor of the ITAPI-G in Experiment 2) probably because, let aside empathy, our participants were always prone to "share" the circle with their best friend as shown by our experiments. 


\section{Conclusion, Limitations, and Further Studies}

Our study confirms that the three most common geometric shapes, the circle, the square and the triangle, are characterized by an expressive halo that influences our decision to associate them with ourselves, our best friend or a stranger with a nonrandom pattern. More specifically, the circle appears to be the most positive shape: with its symmetry, regularity and curvilinearity, it is the geometric shape most frequently associated with the self and the best friend. The square, that is an angular shape with a conformation not as sharp as the triangle is frequently associated to the friend and to the stranger. With this latter identity, the triangle is the shape most frequently chosen together with the square: indeed, according to the relevant literature the triangle is the most negative and threatening shape amongst those used in our experiments. Although our hypotheses involving the interaction between personality traits as aggressivity, social phobia and empathy seemed consistent with such previous findings, we did not confirm any. We point out that few studies have investigated the effect of personality traits on curvature/angularities preferences, finding small or inconsistent results (Cotter et al., 2017).

In general, although it could be hard to disentangle symmetry from contour, that would be an interesting challenge for future works. In fact, the circle is the more symmetrical shape within those used in our experiments, whereas the triangle is the less symmetric and we cannot exclude that the aesthetic preferences conveyed by symmetry, other than from curvature (Corradi \& Munar, 2019), could have played a role in our findings. In fact, symmetry is known to be a pleasant aesthetic feature when it comes to both shapes, objects and faces (Jacobsen et al., 2006; Little et al., 2007). The classic explanation of this preference is based on the evolutionary significance of symmetry, as a cue of health and genetic fitness (Jones et al., 2001; Møller \& Thornhill, 1998). The preference for symmetrical visual stimuli is adaptive, as it would lead to higher quality mate and food choices (Turoman et al., 2018; Watson \& Thornhill, 1994). Although Henderson and colleagues have recently shown that symmetry is only weakly related to perceived health (Henderson et al., 2016), symmetry may remain a positive feature because of its importance for visual processing, facilitating object recognition and detection from 4 months of age (Bornstein et al., 1981; Enquist \& Arak, 1994; Wagemans, 1995).

In addition, future studies could replace the equilateral triangle with a differently shaped or a differently oriented triangle and particularly with a sharper triangle (e.g., isosceles triangle) or with a downward-pointing triangle, the most "threatening" among the tested triangles. If this kind of triangle would arouse more negative emotion due to its similarity with dangerous objects or with the anger expression, maybe also the personality traits could interact with the shape-identity matching. Moreover, it is possible that personality traits actually do not influence the shape-identity associations using a self-reported/explicit task but that they could do it by using more implicit tasks as the Implicit Association Test, or paradigms such as flanker and priming, or the associative learning approach proposed by Sui and coworkers (Sui et al., 2012). Further, it would be interesting to replicate this study using clinical scales (e.g., Minnesota Multiphasic Personality Inventory or the Symptom Checklist-90) to evaluate whether psychiatric symptoms and personality disorders modulate the shape-identity associations.

Finally, we point out that, due to the online distribution of the survey, our samples were not balanced for sex, with a high preponderance of female participants. Despite that, the results of males and females in the shape-identity association task were very similar (see Supplementary materials). We think, however, that deepening sex differences could be suitable and interesting, also considering that personality traits as empathy and aggressivity are usually expressed differently by women and men.

The study of how people associate geometric shapes with the self or with other identities giving them an implicit socioaffective connotation, is interesting for both marketing purposes (Salgado Montejo et al., 2015; Salgado-Montejo et al., 2015), for clinical assessment (Wells, 1950) and in general for all the disciplines interested in the affective processes triggered by visual stimuli.

Supplementary Information The online version contains supplementary material available at https://doi.org/10.1007/s12144-021-02297-z.

Availability of Data and Material The complete dataset is available here: https://doi.org/10.6084/m9.figshare.14363333.v2.

Authors' Contributions VM conceived, designed the experiment and collected, analyzed and interpreted the data. VM wrote the manuscript and LT provided critical revisions and contributed to the final version of the manuscript by reviewing and revising the text. The authors approved the final version for submission and agreed to be accountable to for all aspects of the work.

Funding Open access funding provided by Università degli Studi G. D'Annunzio Chieti Pescara within the CRUI-CARE Agreement. This research did not receive any specific grant from funding agencies in the public, commercial, or not-for-profit sectors.

\section{Declarations}

Ethical Approval All procedures performed in studies involving human participants were in accordance with the ethical standards of the Institutional Review Board of Psychology of the Department of Psychological, Health and Territorial Sciences, and with the 1964 Helsinki declaration and its later amendments or comparable ethical standards. 
Informed Consent Informed consent was obtained from all individual participants included in the study.

Conflict of Interest The authors declare that the research was conducted in the absence of any commercial or financial relationships that could be construed as a potential conflict of interest.

Open Access This article is licensed under a Creative Commons Attribution 4.0 International License, which permits use, sharing, adaptation, distribution and reproduction in any medium or format, as long as you give appropriate credit to the original author(s) and the source, provide a link to the Creative Commons licence, and indicate if changes were made. The images or other third party material in this article are included in the article's Creative Commons licence, unless indicated otherwise in a credit line to the material. If material is not included in the article's Creative Commons licence and your intended use is not permitted by statutory regulation or exceeds the permitted use, you will need to obtain permission directly from the copyright holder. To view a copy of this licence, visit http://creativecommons.org/licenses/by/4.0/.

\section{References}

Aronoff, J. (2006). How we recognize angry and happy emotion in people, places, and things. Cross-Cultural Research, 40(1), 83-105.

Aronoff, J., Woike, B. A., \& Hyman, L. M. (1992). Which are the stimuli in facial displays of anger and happiness? Configurational bases of emotion recognition. Journal of Personality and Social Psychology, 62(6), 1050-1066

Bar, M., \& Neta, M. (2006). Humans prefer curved visual objects. Psychological Science, 17(8), 645-648.

Barrett, L. F., \& Bar, M. (2009). See it with feeling: Affective predictions during object perception. Philosophical transactions of the Royal Society of London. Series B, Biological Sciences, 364(1521), 1325-1334. https://doi.org/10.1098/rstb.2008.0312

Bögels, S. M., Alden, L., Beidel, D. C., Clark, L. A., Pine, D. S., Stein, M. B., \& Voncken, M. (2010). Social anxiety disorder: Questions and answers for the DSM-V. Depression and Anxiety, 27(2), 168-189. https://doi.org/10.1002/da.20670

Bornstein, M. H., Ferdinandsen, K., \& Gross, C. G. (1981). Perception of symmetry in infancy. Developmental Psychology, 17(1), 82-86. https://doi.org/10.1037/0012-1649.17.1.82

Brosch, T., Sander, D., \& Scherer, K. R. (2007). That baby caught my eye... Attention capture by infant faces. Emotion (Washington, D.C.), 7(3), 685-689. https://doi.org/10.1037/1528-3542.7.3.685

Buss, A. H., \& Perry, M. (1992). The aggression questionnaire. Journal of Personality and Social Psychology, 63(3), 452-459. https://doi. org/10.1037//0022-3514.63.3.452

Carbon, C.-C. (2010). The cycle of preference: Long-term dynamics of aesthetic appreciation. Acta Psychologica, 134(2), 233-244. https:// doi.org/10.1016/j.actpsy.2010.02.004

Corradi, G., \& Munar, E. (2019). The Curvature Effect. The Oxford Handbook of Empirical Aesthetics. https://doi.org/10.1093/ oxfordhb/9780198824350.013.24

Cotter, K. N., Silvia, P. J., Bertamini, M., Palumbo, L., \& Vartanian, O. (2017). Curve appeal: Exploring individual differences in preference for curved versus angular objects. I-Perception, 8(2), 2041669517693023. https://doi.org/10.1177/2041669517693023

D’Anselmo, A., Prete, G., Zdybek, P., Tommasi, L., \& Brancucci, A. (2019). Guessing meaning from word sounds of unfamiliar languages: A cross-cultural sound symbolism study. Frontiers in Psychology, 10, 593. https://doi.org/10.3389/fpsyg.2019.00593
Eisenberg, N., \& Fabes, R. A. (1990). Empathy: Conceptualization, measurement, and relation to prosocial behavior. Motivation and Emotion, 14(2), 131-149.

Eisenberg, N., \& Strayer, J. (1987). Critical issues in the study of empathy.

Enquist, M., \& Arak, A. (1994). Symmetry, beauty and evolution. Nature, 372(6502), 169-172. https://doi.org/10.1038/372169a0

Fossati, A., Maffei, C., Acquarini, E., \& Di Ceglie, A. (2003). Multigroup confirmatory component and factor analyses of the Italian version of the aggression questionnaire. European Journal of Psychological Assessment, 19(1), 54-65.

Gable, P. A., \& Harmon-Jones, E. (2008). Approach-motivated positive affect reduces breadth of attention. Psychological Science, 19(5), 476-482. https://doi.org/10.1111/j.1467-9280.2008.02112.x

Gronau, N., Cohen, A., \& Ben-Shakhar, G. (2003). Dissociations of personally significant and task-relevant distractors inside and outside the focus of attention: A combined behavioral and psychophysiological study. Journal of Experimental Psychology: General, 132(4), 512-529.

Henderson, A. J., Holzleitner, I. J., Talamas, S. N., \& Perrett, D. I. (2016). Perception of health from facial cues. Philosophical Transactions of the Royal Society B: Biological Sciences, 371(1693), 20150380. https://doi.org/10.1098/rstb.2015.0380

Hoffman, M. L. (1987). The contribution of empathy to justice and moral judgment. Empathy and its development, 4780.

Hogarth, W. (1753). The analysis of beauty: Written with a view of fixing the fluctuating ideas of taste. Georg Olms Verlag.

Huesmann, L. R. (1994). Aggressive behavior: Current perspectives. Springer Science \& Business Media.

Jacobsen, T., Schubotz, R. I., Höfel, L., \& Cramon, D. Y. V. (2006). Brain correlates of aesthetic judgment of beauty. NeuroImage, 29(1), 276-285. https://doi.org/10.1016/j.neuroimage.2005.07.010

Jones, B. C., Little, A. C., Penton-Voak, I. S., Tiddeman, B. P., Burt, D. M., \& Perrett, D. I. (2001). Facial symmetry and judgements of apparent health: Support for a "good genes" explanation of the attractiveness-symmetry relationship. Evolution and Human Behavior, 22(6), 417-429. https://doi.org/10.1016/S1090-5138(01) 00083-6

Köhler, W. (1970). Gestalt psychology: An introduction to new concepts in modern psychology (Vol. 18). WW Norton \& Company.

Larson, C. L., Aronoff, J., \& Steuer, E. L. (2012). Simple geometric shapes are implicitly associated with affective value. Motivation and Emotion, 36(3), 404-413. https://doi.org/10.1007/s11031-0119249-2

Leder, H., \& Carbon, C.-C. (2005). Dimensions in appreciation of car interior design. Applied Cognitive Psychology, 19(5), 603-618. https://doi.org/10.1002/acp.1088

Little, A. C., Apicella, C. L., \& Marlowe, F. W. (2007). Preferences for symmetry in human faces in two cultures: Data from the UK and the Hadza, an isolated group of hunter-gatherers. Proceedings of the Royal Society B: Biological Sciences, 274(1629), 3113-3117. https://doi.org/10.1098/rspb.2007.0895

Lundholm, H. (1921). The affective tone of lines: Experimental researches. Psychological Review, 28(1), 43-60. https://doi.org/10. 1037/h0072647

Mehrabian, A. (1996). Manual for the balanced emotional empathy scale (BEES). Available from Albert Mehrabian, 1130.

Meneghini, A. M., Sartori, R., \& Cunico, L. (2006). Adattamento e validazione su campione italiano della Balanced Emotional Empathy Scale di A. Mehrabian. Ricerche di Psicologia.

Møller, A. P., \& Thornhill, R. (1998). Bilateral symmetry and sexual selection: A Meta-analysis. The American Naturalist, 151(2), 174 192. https://doi.org/10.1086/286110

Ohman, A., \& Mineka, S. (2001). Fears, phobias, and preparedness: Toward an evolved module of fear and fear learning. 
Psychological Review, 108(3), 483-522. https://doi.org/10.1037/ 0033-295x.108.3.483

Osgood, C. E., Suci, G. J., \& Tannenbaum, P. H. (1957). The measurement of meaning. University of Illinois Press.

Palermo, R., \& Rhodes, G. (2007). Are you always on my mind? A review of how face perception and attention interact. Neuropsychologia, 45(1), 75-92. https://doi.org/10.1016/j. neuropsychologia.2006.04.025

Pavlova, M., Sokolov, A. A., \& Sokolov, A. (2005). Perceived dynamics of static images enables emotional attribution. Perception, 34(9), $1107-1116$

Perussia, F. (2006). ITAPI-G: Manuale: inventario italiano di personalità, Italia personality inventory, forma $\mathrm{G}$ (generale). Unicopli.

Raulin, M. L., \& Wee, J. L. (1984). The development and initial validation of a scale to measure social fear. Journal of Clinical Psychology, 40(3), 780-784. https://doi.org/10.1002/10974679(198405)40:3<780::aid-jclp2270400324>3.0.co;2-m

Reber, R., Schwarz, N., \& Winkielman, P. (2004). Processing fluency and aesthetic pleasure: Is beauty in the Perceiver's processing experience? Personality and Social Psychology Review, 8(4), 364-382. https://doi.org/10.1207/s15327957pspr0804 3

Ro, T., Russell, C., \& Lavie, N. (2001). Changing faces: A detection advantage in the flicker paradigm. Psychological Science, 12(1), 94-99. https://doi.org/10.1111/1467-9280.00317

Salgado Montejo, A., Alvarado, J. A., Velasco, C., Salgado, C. J., Hasse, K., \& Spence, C. (2015). The sweetest thing: The influence of angularity, symmetry, and the number of elements on shape-valence and shape-taste matches. Frontiers in Psychology, 6, 1382.

Salgado-Montejo, A., Tapia Leon, I., Elliot, A. J., Salgado, C. J., \& Spence, C. (2015). Smiles over frowns: When curved LINES influence Product preference: LINES AND PRODUCT PREFERENCE. Psychology \& Marketing, 32(7), 771-781. https://doi.org/10.1002/ mar.20817

Sapir, E. (1929). A study in phonetic symbolism. Journal of Experimental Psychology, 12(3), 225-239.

Silvia, P. J., \& Barona, C. M. (2009). Do people prefer curved objects? Angularity, expertise, and aesthetic preference. Empirical Studies of the Arts, 27(1), 25-42.

Stopa, L., Denton, R., Wingfield, M., \& Taylor, K. N. (2013). The fear of others: A qualitative analysis of interpersonal threat in social phobia and paranoia. Behavioural and Cognitive Psychotherapy, 41(2), 188-209. https://doi.org/10.1017/S1352465812000422
Sui, J., He, X., \& Humphreys, G. W. (2012). Perceptual effects of social salience: Evidence from self-prioritization effects on perceptual matching. Journal of Experimental Psychology: Human Perception and Performance, 38(5), 1105-1117.

Sui, J., \& Liu, C. H. (2009). Can beauty be ignored? Effects of facial attractiveness on covert attention. Psychonomic Bulletin \& Review, 16(2), 276-281. https://doi.org/10.3758/PBR.16.2.276

Turoman, N., Velasco, C., Chen, Y.-C., Huang, P.-C., \& Spence, C. (2018). Symmetry and its role in the crossmodal correspondence between shape and taste. Attention, Perception, \& Psychophysics, 80(3), 738-751. https://doi.org/10.3758/s13414-017-1463-x

Velasco, C., Salgado-Montejo, A., Elliot, A. J., Woods, A. T., Alvarado, J., \& Spence, C. (2016). The shapes associated with approach/ avoidance words. Motivation and Emotion, 40(5), 689-702.

Vuilleumier, P. (2005). How brains beware: Neural mechanisms of emotional attention. Trends in Cognitive Sciences, 9(12), 585-594. https://doi.org/10.1016/j.tics.2005.10.011

Wagemans, J. (1995). Detection of visual symmetries. Spatial Vision, 9(1), 9-32. https://doi.org/10.1163/156856895X00098

Watson, D. G., Blagrove, E., Evans, C., \& Moore, L. (2012). Negative triangles: Simple geometric shapes convey emotional valence. Emotion, 12(1), 18-22.

Watson, P. J., \& Thornhill, R. (1994). Fluctuating asymmetry and sexual selection. Trends in Ecology \& Evolution, 9(1), 21-25. https://doi. org/10.1016/0169-5347(94)90227-5

Wells, F. L. (1950). Some projective functions of simple geometrical figures: Cases LXXXV-XCV. The Pedagogical Seminary and Journal of Genetic Psychology, 77(2), 187-210. https://doi.org/10. 1080/08856559.1950.10533548

Westbury, C. (2005). Implicit sound symbolism in lexical access: Evidence from an interference task. Brain and Language, 93(1), 10-19. https://doi.org/10.1016/j.bandl.2004.07.006

Wrangham, R. W. (2018). Two types of aggression in human evolution. Proceedings of the National Academy of Sciences of the United States of America, 115(2), 245-253. https://doi.org/10.1073/pnas. 1713611115

Publisher's Note Springer Nature remains neutral with regard to jurisdictional claims in published maps and institutional affiliations. 